\title{
Pantothenate kinase-associated neurodegeneration
}

INSERM

\section{Source}

INSERM. (1999). Orphanet: an online rare disease and orphan drug data base.

Pantothenate kinase-associated neurodegeneration. ORPHA:157850

Pantothenate kinase-associated neurodegeneration (PKAN) is the most common type of neurodegeneration with brain iron accumulation (NBIA; see this term), a rare neurodeg enerative disorder characterized by progressive extrapyramidal dysfunction (dystonia, rigidity, choreoathetosis), iron accumulation on the brain and axonal spheroids in the central nervous system. 